Research Article

\title{
Effects of Renal Denervation via Renal Artery Adventitial Cryoablation on Atrial Fibrillation and Cardiac Neural Remodeling
}

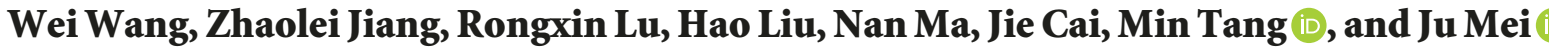 \\ Department of Cardiothoracic Surgery, Xinhua Hospital, Shanghai Jiaotong University School of Medicine, \\ Shanghai 200092, China \\ Correspondence should be addressed to Min Tang; tangmin@xinhuamed.com.cn and Ju Mei; meiju@xinhuamed.com.cn
}

Received 20 August 2018; Accepted 2 October 2018; Published 11 December 2018

Guest Editor: Tong Liu

Copyright $(2018$ Wei Wang et al. This is an open access article distributed under the Creative Commons Attribution License, which permits unrestricted use, distribution, and reproduction in any medium, provided the original work is properly cited.

\begin{abstract}
Introduction. Catheter-based renal denervation (RDN) could reduce cardiac sympathetic nerve activity (SNA) and inhibit atrial fibrillation (AF). However, the reliability is uncertain, because the renal sympathetic nerves are mainly distributed in the adventitial surface of the renal artery. Objective. The aims of this study were to test the hypothesis that renal artery adventitial ablation (RAAA) definitely had the effects of RDN and to study the effects of RDN via renal artery adventitial cryoablation (RAAC) on AF and cardiac neural remodeling. Methods. Twenty beagle canines were randomly assigned to two groups: the left RDN group (LRDN, $n=10$ ), which underwent left RDN via RAAC; the Sham group $(n=10)$. After 2 months of postoperative recovery, AF vulnerability, AF duration, and histological examination were performed in both groups. Results. Compared with the Sham group, left stellate ganglion (LSG) tissue fibrosis was increased in the LRDN group. LRDN significantly increased the percentage of TH-negative ganglionic cells and decreased the density of TH-positive nerves in the LSG $(P<0.001)$. Also, the densities of TH-positive nerves and GAP43 immunoreactivity within the left atrium (LA) were significantly decreased in the LRDN group $(P<0.05)$. After LA burst pacing, all 10 canines $(100 \%)$ could be induced AF in the Sham group, but only 4 of 10 canines $(40 \%)$ could be induced AF in the LRDN group $(P=0.011)$. The percentage of LA burst stimulation with induced AF was $26.7 \%$ (8/30) in the LRDN group, which was significantly decreased compared with that of the Sham group $(53.3 \%, 16 / 30)(P=0.035)$. In addition, AF duration was also significantly decreased in the LRDN group (13.3 $\pm 5.1 \mathrm{~s})$ compared with that of the Sham group $(20.3 \pm 7.3 \mathrm{~s}, P=0.024)$. Conclusions. RDN via RAAC could cause cardiac neural remodeling and effectively inhibit AF inducibility and shorten AF duration. It may be useful in selecting therapeutic approaches for AF patients.
\end{abstract}

\section{Introduction}

Atrial fibrillation (AF) is the most common cardiac arrhythmia [1]. Autonomic neural system (ANS) activation played a key role in the occurrence and maintenance of AF, which could induce atrial structural remodeling and the changes of atrial electrophysiology. Reasonable autonomic nerve intervention could reduce cardiac sympathetic nerve activity (SNA) and improve the treatment of AF [2-4]. Several studies have showed that catheter-based renal denervation (RDN) could reduce cardiac SNA and inhibit AF
[5-7]. Linz et al. [5] demonstrated that catheter-based RDN could reduce atrial sympathetic nerve sprouting, structural alterations, and $\mathrm{AF}$ complexity in goats with persistent $\mathrm{AF}$. Wang et al. [6] found that catheter-based RDN could inhibit the progression of paroxysmal $\mathrm{AF}$ by reducing the incidences of AF and shortening the duration of AF. However, the reliability of catheter-based RDN is uncertain, because the renal sympathetic nerves are mainly distributed in the adventitial surface of the renal artery $[8,9]$. We speculated that renal artery adventitial ablation (RAAA) may have better effects of RDN than catheter-based RDN. At present, 
epicardial cryoablation has been widely used in the surgical AF ablation, which could achieve satisfactory integrity and transmurality of ablation lines. Therefore, we aimed to perform this study as following: (1) to test the hypothesis that RAAA definitely had the effects of RDN; (2) to study the effects of RDN via renal artery adventitial cryoablation (RAAC) on AF and cardiac neural remodeling.

\section{Methods}

2.1. Animals. This study was approved by the Ethics Committee of Xinhua Hospital, Shanghai Jiao Tong University, School of Medicine. All animal experiments were performed in accordance with the National Institutes of Health Guide for the Care and Use of Laboratory Animals. A total of 20 beagle canines (male, $15-20 \mathrm{~kg}$ ) were studied in the Experimental Animal Center of Xinhua Hospital. The 20 beagle canines were randomly assigned to the following two groups: (1) the left RDN group (LRDN, $n=10$ ), which underwent left RDN via RAAC; (2) the Sham group $(n=10)$.

2.2. Renal Artery Adventitial Ablation (RAAA). Routine clinical monitoring was performed during the surgical procedure. Anesthesia was induced with ketamine (5$10 \mathrm{mg} / \mathrm{kg}$ ) and midazolam (0.1-0.2 mg/kg IV). After intubation and mechanical ventilation, anesthesia was maintained with $2.0 \%$ isoflurane. The canines were placed in the right lateral decubitus position. A 6 to $8 \mathrm{~cm}$ subcostal incision was made on the abdomen to separate and expose the left renal artery (LRA). The proximal renal artery close to the renal artery ostium was subjected to cryoablation (both ventral side and dorsal side of the renal artery, cryoablation temperature $-70^{\circ} \mathrm{C}, 120$ seconds for each side) using a cryoprobe $(5 \mathrm{~cm}$ in length, with a diameter of $6 \mathrm{~mm}$ on the tip, CryoICE, Atricure, USA) (Figure 1(a)). The incision was then closed. Cefuroxime sodium $(30 \mathrm{mg} / \mathrm{kg})$ was administered by intravenous perfusion during the surgery and used for three days after the surgery.

RAAC was performed for all canines in the LRDN group. For the Sham group, we only separated and exposed the LRA through the same incision, but RAAC was not performed.

2.3. AF Vulnerability Studies. After 2 months of postoperative recovery, electrophysiology experiment was performed. After the canines were anesthetized, the tips of two pairs of looping electrodes were directly sutured to the surface of the left atrial appendage (LAA) through the left third intercostal incision. One pair of looping electrodes was used to record the left atrial (LA) electric signal, and the other pair of looping electrodes was used for pacing. The electrocardiograms and electric signals were recorded simultaneously using the LabChart system (ADInstruments, AUS). Continuous 6 hours' rapid atrial pacing (RAP) (600 bpm, $0.5 \mathrm{~ms}$, twice threshold current) was administered at the LA site for all canines. After that, AF inducibility was assessed by the burst pacing protocol at the LA site. Burst pacing was performed for each canine, at a cycle length of $50 \mathrm{~ms}$ and a stimulus output of $0.5 \mathrm{~V}$ plus twice threshold current for $60 \mathrm{~s}$. Signals were sampled at $2 \mathrm{kHz}$ and stored with the LabChart system. AF was defined as irregular atrial rates $>500 \mathrm{bpm}$ and lasting over $5 \mathrm{~s}$ associated with irregular atrioventricular conduction. AF inducibility was repeated for 3 times by burst pacing.

2.4. Histologic Analysis. After the electrophysiology experiment, the canines were then euthanized, and the tissues of LRA, left stellate ganglion (LSG), and LA were harvested. Small portions of the tissues were fixed in $4 \%$ formalin for $45 \mathrm{~min}$ and then stored in $70 \%$ alcohol for analysis. The tissues were paraffin embedded and cut into $5 \mu \mathrm{m}$ thick sections routinely. The LRA was stained with haematoxylin-eosin (HE). Immunohistochemical staining of the LSG was performed using an anti-tyrosine hydroxylase- (TH-) antibody (22941, Immunostar, USA). Immunohistochemical staining of the LA was performed using an anti-TH-antibody (22941, Immunostar, USA) and an anti-growth-associated protein 43- (GAP-43-) antibody (NB300-143SS, Novus, USA). LSG tissues were also stained with the Masson trichrome stain. All slides were examined manually under a DP72 microscope (Olympus, Tokyo, Japan).

2.5. Statistical Analysis. The software SPSS 22.0 (SPSS, USA) was used for the statistical analysis. Continuous variables were expressed as mean \pm standard deviation (SD) and $95 \%$ confidence interval (CI). Student's $t$ test was used to compare continuous variables between the two groups. Categorical variables were presented as frequencies and proportions. The chi-square test or Fisher's exact test was used to compare categorical variables. A $P$ value of $<0.05$ was considered to be significant for these comparisons.

\section{Results}

3.1. Effects of LRDN on the Morphological Changes of LRA. After 2 months of postoperative recovery, the canines were sacrificed and LRA was harvested. Figure 1(b) shows the morphological features of the LRA with H\&E staining at 2 months after RAAC. The adventitia, media, and intima of renal artery wall were injured by RAAC, which displayed as the damaged region (DR). Compared with the normal region (NR), neointima formation (yellow arrow head) could be found corresponding to the DR. These features of the LRA demonstrated that RAAC could create transmural ablation from the adventitia to the intima of renal artery.

3.2. Effects of LRDN on Neural Remodeling of LSG. Figure 2 shows typical examples of LSG structural remodeling in both the Sham group and the LRDN group. Compared with the Sham group (Figures 2(a) and 2(b)), LSG tissue fibrosis was increased in the LRDN group (Figures 2(c) and $2(\mathrm{~d}))$.

Figure 3 shows TH staining of the LSG in both the Sham group (Figure $3(\mathrm{a})$ ) and the LRDN group (Figure $3(\mathrm{~b})$ ). 


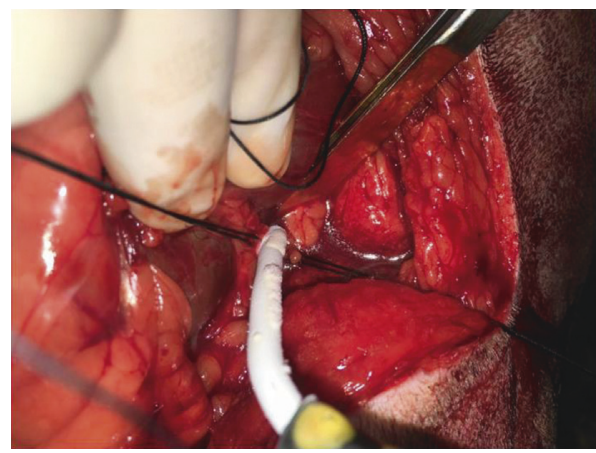

(a)

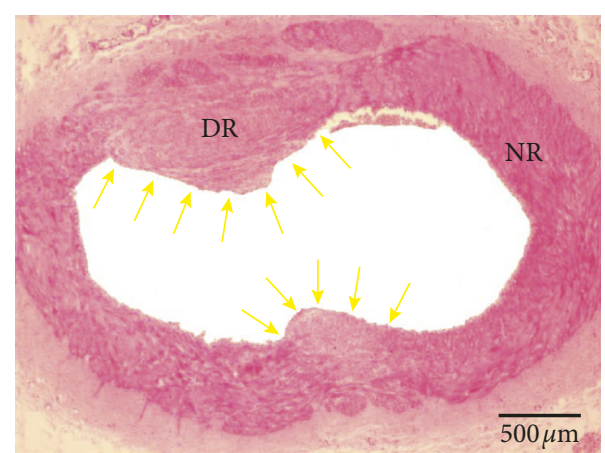

(b)

FIGURE 1: Renal artery ablation. (a) Renal artery adventitial cryoablation (RAAC). (b) Morphological changes of the LRA after LRDN. DR: damage region; NR: normal region.

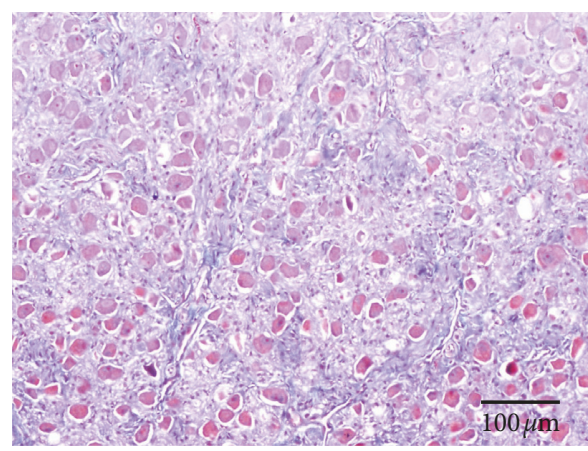

(a)

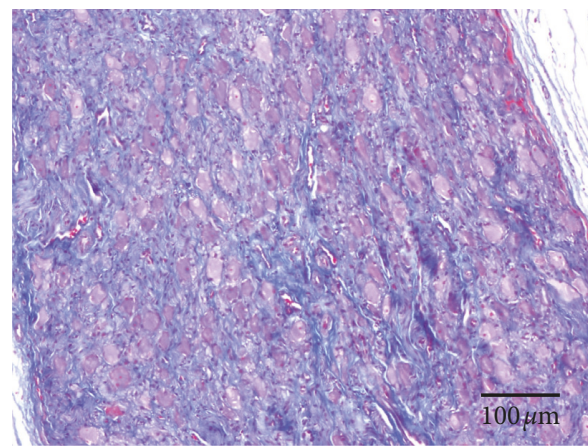

(c)

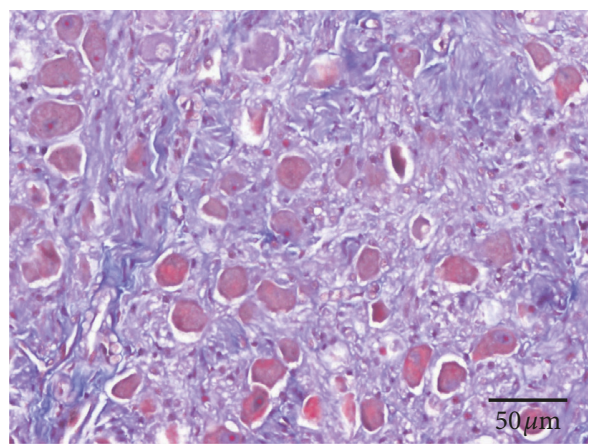

(b)

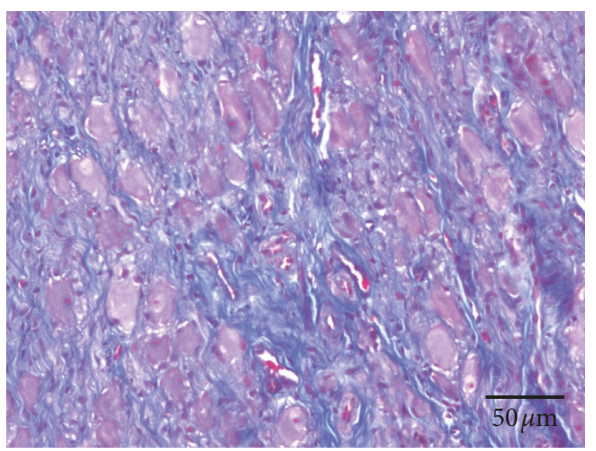

(d)

FIGURE 2: Masson trichrome staining of LSG. (a) and (b): Tissue fibrosis seen at low magnification in Sham group; (c) and (d): Tissue fibrosis seen at high magnification in LRDN group.

Compared with that of the Sham group, LRDN significantly increased the percentage of TH-negative ganglionic cells in the LSG in canines of the LRDN group. The mean percentage of TH-negative ganglionic cells in canines of the LRDN group (9.2 $\pm 1.7 \%$ (95\% CI, $8.2 \%$ to $10.3 \%)$ ) was significantly higher than that in the Sham group (4.6 $\pm 1.9 \%$ (95\% CI, $3.4 \%$ to $5.8 \%))(P<0.001$, Figure $4(\mathrm{a}))$. For canines with LRDN, there was a significantly decreased density of THpositive nerves in the LSG $\left(116835.8 \pm 15794.8 \mu \mathrm{m}^{2} / \mathrm{mm}^{2}\right.$ (95\% CI, 106510.7 to 125826.5)) compared to the canines of the Sham group $\left(169784.5 \pm 21284.6 \mu \mathrm{m}^{2} / \mathrm{mm}^{2}\right.$ (95\% CI, 156184.9 to 182772.4$))(P<0.001$, Figure $4(\mathrm{~b}))$.
3.3. Effects of LRDN on Neural Remodeling of LA. Figure 5 compares the results of immunostaining of TH and GAP43 in the LA between the Sham group and the LRDN group. The nerve density of LA for each group was expressed as a mean of nerve densities. Compared with the Sham group (Figure 5(a), $1660.6 \pm 468.4 \mu \mathrm{m}^{2} / \mathrm{mm}^{2}$ (95\% CI, 1385.5 to 1958.3)), the densities of TH-positive nerves within the LA were significantly decreased in the LRDN group (Figure 5(b), $1233.4 \pm 345.4 \mu \mathrm{m}^{2} / \mathrm{mm}^{2}$ (95\% CI, 1032.4 to 1455.7$)$ ) $(P=0.032$, Figure $4(c))$. The density of GAP43 immunoreactivity in the LA was $\left(2313.9 \pm 411.0 \mu \mathrm{m}^{2} / \mathrm{mm}^{2}(95 \% \mathrm{CI}\right.$, 2048.4 to 2560.6)) in the Sham group (Figure 5(c)), which was 


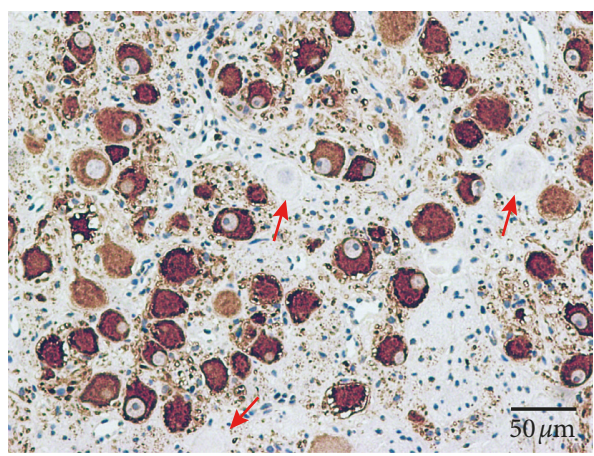

(a)

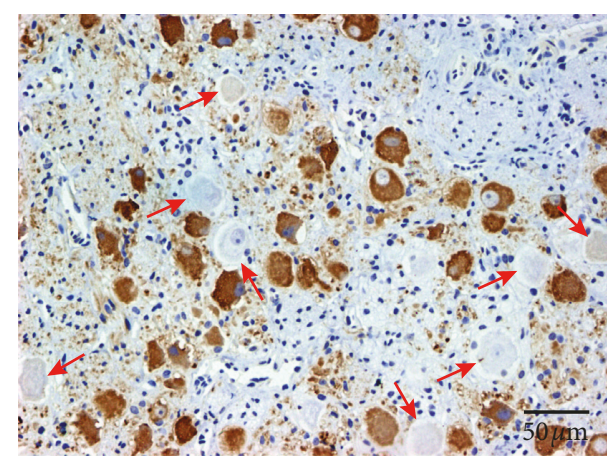

(b)

Figure 3: Tyrosine hydroxylase (TH) immunostaining of LSG. (a) TH staining showed TH-negative ganglion cells (red arrows) and THpositive ganglion cells in LSG in Sham group; (b) TH staining showed TH-negative ganglion cells (red arrows) and TH-positive ganglion cells in LSG in LRDN group.

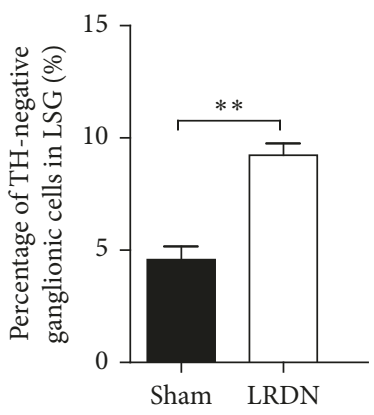

(a)

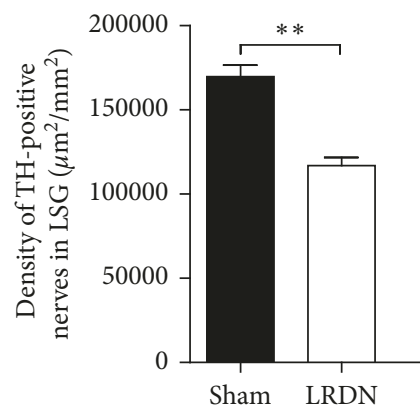

(b)

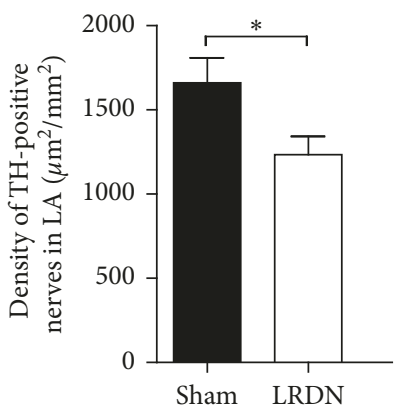

(c)

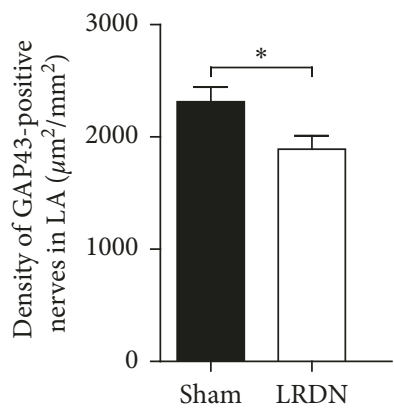

(d)

FIGURE 4: Immunostaining results in LSG or LA between Sham group and LRDN group. (a) The percentage of TH-negative ganglionic cells in LSG in both Sham group and LRDN group; (b) comparison between the density of TH-positive nerves in LSG between Sham group and LRDN group; (c) the density of TH-positive nerves in LA in both Sham group and LRDN group; (d) comparison between the density of GAP43-positive nerves in LA between Sham group and LRDN group $\left({ }^{*} P<0.05\right.$ vs. the Sham group; ${ }^{* *} P<0.01$ vs. the Sham group).

significantly higher than that of the LRDN group (Figure 5(d), $1890.9 \pm 383.8 \mu \mathrm{m}^{2} / \mathrm{mm}^{2}(95 \% \mathrm{CI}, 1648.9$ to 2115.1$\left.)\right)$ $(P=0.029$, Figure $4(\mathrm{~d}))$.

\subsection{Effects of LRDN on AF Inducibility and AF Duration.} After LA burst pacing, all 10 canines (100\%) could be induced AF in the Sham group, but only 4 of 10 canines (40\%) could be induced AF in the LRDN group $(P=0.011$, Figure $6(\mathrm{a}))$. The percentage of LA burst stimulation with induced AF was $26.7 \%$ (8/30) in the LRDN group, which was significantly decreased compared with that of the Sham group $(53.3 \%, 16 / 30)(P=0.035$, Figure $6(\mathrm{~b}))$. In addition, AF duration was also significantly decreased in the LRDN group $(13.3 \pm 5.1 \mathrm{~s}, 95 \%$ CI 10.2 17.2) compared with that of the Sham group $(20.3 \pm 7.3$ s, 95\% CI 16.9 24.0, $P=0.024$, Figure 6(c)).

\section{Discussion}

ANS activation could induce significant and heterogeneous changes of atrial electrophysiology. Multiple evidences have demonstrated that ANS, especially sympathetic nervous system, may play a key role in the occurrence and maintenance of AF $[10,11]$. In anatomical structure, the heart is innervated by the extrinsic nervous system and the intrinsic nervous system. Both the extrinsic and intrinsic cardiac nervous systems are important for arrhythmogenesis, such as $\mathrm{AF}[2,12]$. The extrinsic cardiac nervous system mainly includes stellate ganglion (SG) and vagal nerve. The intrinsic cardiac nerves are mostly found in the atrial wall. Neuromodulation methods that reduce sympathetic nerve activity may be helpful in controlling $\operatorname{AF}[13,14]$. In the previous study, Shen et al. has reported that vagal nerve stimulation (VNS) could effectively suppress SGNA and reduce the incidences of paroxysmal atrial tachyarrhythmias (PAT) [15]. Therefore, the modulation of ANS may be a promising target for intervention in AF patients. At present, ganglionated plexi (GP) ablation has been used to cure AF in patients [16-18]. However, nerve regeneration after GP ablation is still related to AF recurrence $[19,20]$.

In order to improve the effects of ANS modulation, several methods have been developed. RDN was considered to be one of the recommended methods to modulate ANS. Catheter-based approach has been developed for RDN, which has been proved to be able to reduce cardiac SNA and 


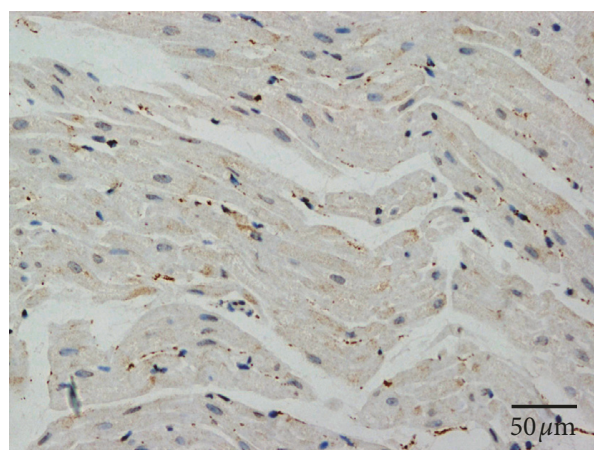

(a)

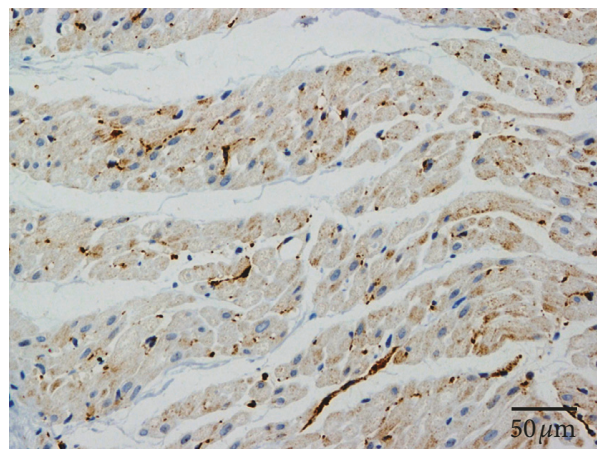

(c)

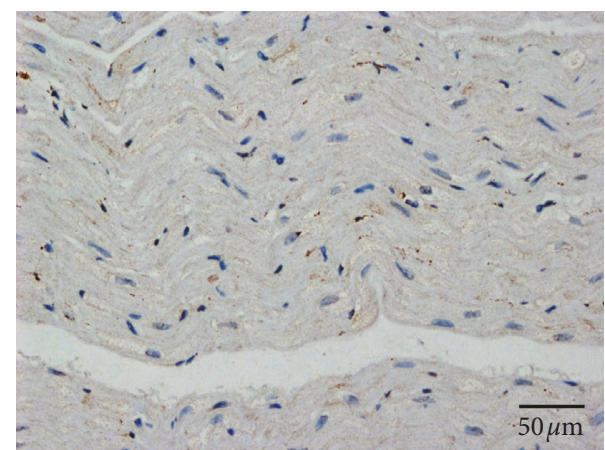

(b)

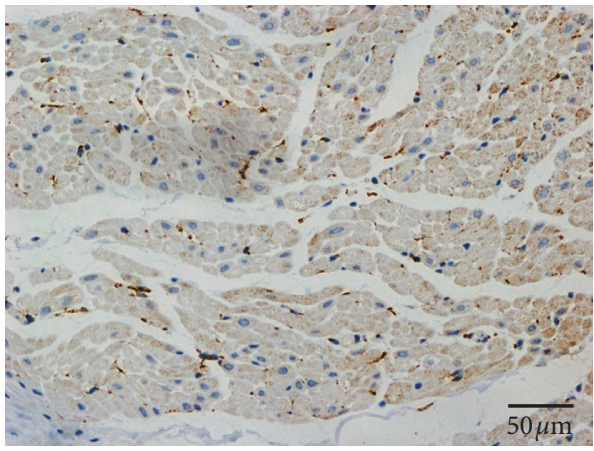

(d)

FIgURE 5: TH and GAP43 immunostaining of LA. (a) TH staining showed the densities of TH-positive nerves (brown) within the LA in Sham group; (b) TH staining showed the densities of TH-positive nerves within the LA in LRDN group; (c) GAP43 staining showed the density of GAP43 immunoreactivity in the LA in Sham group; (d) GAP43 staining showed the density of GAP43 immunoreactivity in the LA in LRDN group.

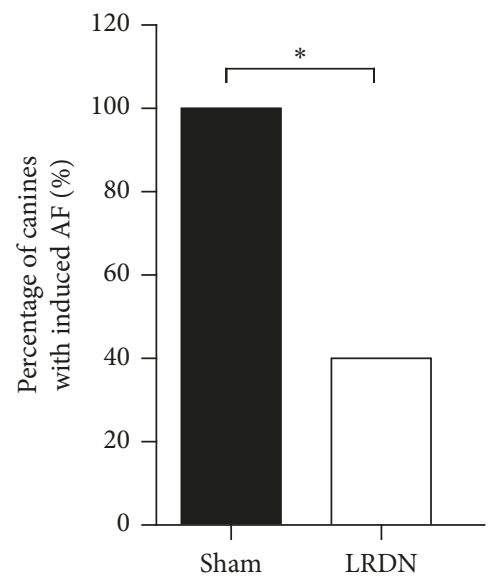

(a)

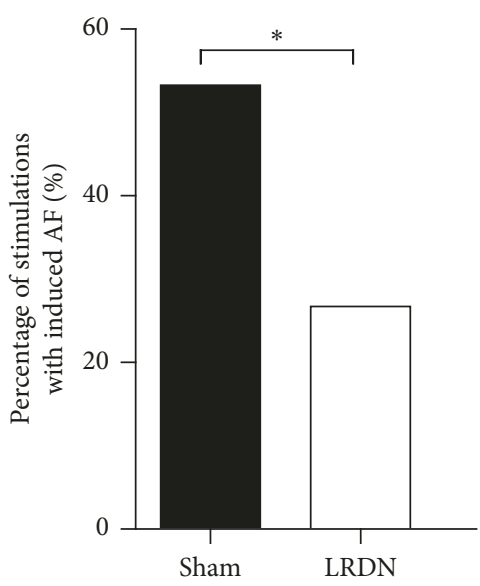

(b)

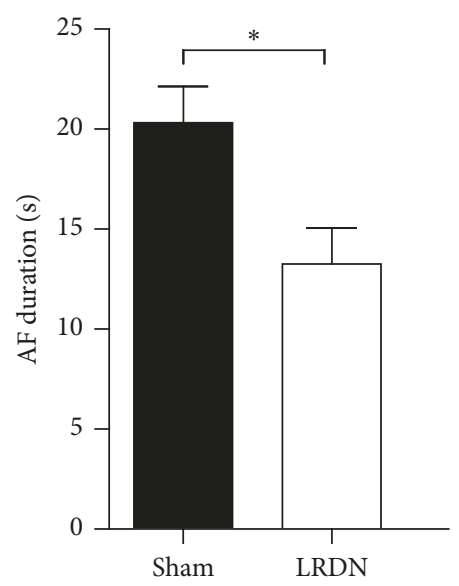

(c)

FIGURE 6: AF inducibility and AF duration between Sham group and LRDN group. (a) The percentage of canines with induced AF in LSG in both Sham group and LRDN group; (b) comparison between the percentage of stimulations with induced AF between Sham group and LRDN group; (c) comparison between the AF duration between Sham group and LRDN group $\left({ }^{*} P<0.05\right.$ vs. the Sham group).

inhibit AF [21]. However, the outcomes of catheter-based RDN are still uncertain, because the afferent and efferent nerves are mainly distributed in the adventitia of the renal artery $[8,9]$. In this study, we performed RDN via RAAC (both ventral side and dorsal side of the renal artery, cryoablation temperature $-70^{\circ} \mathrm{C}, 120$ seconds for each side).
LRA with H\&E staining showed that the adventitia, media, and intima of renal artery wall were injured by RAAC, which demonstrated that RAAC could create transmural ablation of renal artery for RDN.

In the previous studies, Huang et al. demonstrated that 3 hours of left-sided electrical stimulation of renal sympathetic 
nerve was able to increase both systemic and cardiac sympathetic nerve activities and cause neural remodeling in the LSG, which can be proarrhythmic in dogs in the presence of acute myocardial infarction. It suggested that there were direct or indirect connections between renal sympathetic nerve and LSG $[22,23]$. Tsai et al. founded that RDN could reduce SG nerve activity and cause sympathetic nerve remodeling. His findings in part certificated the connections between renal sympathetic nerve and LSG [24]. According to these previous reports, increasing renal SNA could increase SG nerve activity and cause corresponding SG neural remodeling, whereas decreasing renal SNA could decrease SG nerve activity and cause corresponding SG neural remodeling. In our study, we also found that RDN via RAAC could increase LSG tissue fibrosis, increase the percentage of $\mathrm{TH}$-negative ganglionic cells, and significantly decrease the density of TH-positive nerves in the LSG. That is, sympathetic components in LSG were significantly reduced by RDN via RAAC. Therefore, our findings demonstrated that RAAC definitely had the effects of RDN and inhibited SG nerve activity.

SG is the sympathetic ganglion formed by fusion of the inferior cervical ganglion and the first thoracic ganglion. SG is considered to be an important source of cardiac sympathetic innervation. It gave rise to sympathetic nerves that innervate atrium and ventricle $[25,26]$. Cao et al. induced cardiac sympathetic nerve sprouting by infusing nerve growth factor (NGF) to the LSG in dogs with myocardial infarction and complete atrioventricular block [27]. This demonstrated that neural remodeling of intrinsic cardiac nerve was related to the extrinsic cardiac nerve. In our study, we harvested the LA tissues and examined with immunostaining of TH and GAP43. Both the density of TH-positive nerve and GAP43 immunoreactivity within the LA were significantly decreased in the LRDN group compared with that of the Sham group. Our findings demonstrated that LRDN not only significantly decreased the components of sympathetic nerve but also significantly inhibited novel sympathetic nerve sprouting in LA.

Overactivity of the sympathetic nervous system played an important role in the occurrence and maintenance of AF. Increased SNA or atrial sympathetic innervation is associated with increased incidence and duration of AF. In chronic AF patients, atrial sympathetic nerve densities are also significantly increased $[28,29]$. Now, it was suggested that RDN could reduce SNA and decrease susceptibility to AF [5-7]. In our study, we also examined the effects of LRDN on AF inducibility and AF duration. Compared with the Sham group (100\%), only $40 \%$ canines could be induced AF by LA burst pacing in the LRDN group. The percentage of LA burst stimulation with induced AF was $26.7 \%$ in the LRDN group, which was significantly lower than that of the Sham group (53.3\%). Besides, LRDN also significantly decreased AF duration. Our data confirmed that RDN via RAAC could effectively inhibit AF inducibility and shorten AF duration.

\section{Clinic Implications}

The present study showed that RAAC could achieve the effects of RDN and inhibit cardiac SNA. Compared with the
Sham group, RDN via RAAC could effectively inhibit AF inducibility and shorten AF duration. Therefore, we conclude that additional RDN via RAAC may be able to inhibit the occurrence and maintenance of $\mathrm{AF}$ and improve the therapeutic effects of AF.

\section{Limitations}

The present study has few limitations. Firstly, we did not evaluate the SG function by directly recording SG nerve activity, which may reflect SG function more objectively. Secondly, we only performed left side RDN. The reason is that previous studies $[22,23]$ have demonstrated that leftsided electrical stimulation of renal sympathetic nerve was able to increase both systemic and cardiac sympathetic activity and cause neural remodeling in LSG. We guess that LRDN may be able to decrease systemic and cardiac SNA. So, we would like to examine the effects of LRDN by this study. Thirdly, we got satisfactory short-term effects of RDN, but the midterm or long-term effects were not observed. The midterm or long-term effects of RDN via RAAC remain to be explored in the future. Fourthly, RDN via adventitial ablation may be potentially better than catheter-based RDN, but $\mathrm{RDN}$ via adventitial ablation have more trauma than that of catheter-based RDN.

\section{Conclusions}

Renal artery adventitial ablation (RAAA) definitely had the effects of RDN. RDN via renal artery adventitial cryoablation (RAAC) could cause cardiac neural remodeling and effectively inhibit $\mathrm{AF}$ inducibility and shorten $\mathrm{AF}$ duration. It may be useful in selecting therapeutic approaches for AF patients.

\section{Data Availability}

The data used to support the findings of this study are available from the corresponding author upon request.

\section{Conflicts of Interest}

There are no conflicts of interest to report.

\section{Authors' Contributions}

Wei Wang and Zhaolei Jiang contributed equally to this work.

\section{Acknowledgments}

We are grateful for the financial support from National Key Clinical Specialty, National Natural Science Foundation of China (Grant Nos. 81570290 and 81600264), Shanghai Science and Technology Grant (Grant No. 15411952600), Shanghai Shenkang Hospital Development Center Grant (Grant No. 16CR3087B), Shanghai Xinhua Hospital Grant (Grant No. 15YJ13), and Shanghai Young Physician Training Program. 


\section{References}

[1] Y. Guo, Y. Tian, H. Wang, Q. Si, Y. Wang, and G. Y. H. Lip, "Prevalence, incidence, and lifetime risk of atrial fibrillation in China: new insights into the global burden of atrial fibrillation," Chest, vol. 147, no. 1, pp. 109-119, 2015.

[2] M. J. Shen and D. P. Zipes, "Role of the autonomic nervous system in modulating cardiac arrhythmias," Circulation Research, vol. 114, no. 6, pp. 1004-1021, 2014.

[3] M. J. Shen, E. K. Choi, A. Y. Tan et al., "Neural mechanisms of atrial arrhythmias," Nature Reviews Cardiology, vol. 9, no. 1, pp. 30-39, 2011.

[4] J. V. Jayachandran, H. J. Sih, W. Winkle, D. P. Zipes, G. D. Hutchins, and J. E. Olgin, "Atrial fibrillation produced by prolonged rapid atrial pacing is associated with heterogeneous changes in atrialsympathetic innervation," Circulation, vol. 101, no. 10, pp. 1185-1191, 2000.

[5] D. Linz, A. van Hunnik, M. Hohl et al., "Catheter-based renal denervation reduces atrial nerve sprouting and complexity of atrial fibrillation in goats," Circulation: Arrhythmia and Electrophysiology, vol. 8, no. 2, pp. 466-474, 2015.

[6] X. Wang, C. Huang, Q. Zhao et al., "Effect of renal sympathetic denervation on the progression of paroxysmal atrial fibrillation in canines with long-term intermittent atrial pacing," Europace, vol. 17, no. 4, pp. 647-654, 2015.

[7] Q. Zhou, X. Zhou, Z. L. TuEr-Hong et al., "Renal sympathetic denervation suppresses atrial fibrillation induced by acute atrial ischemia/infarction through inhibition of cardiac sympathetic activity," International Journal of Cardiology, vol. 203, pp. 187-195, 2016.

[8] D. L. Bhatt, D. E. Kandzari, W. W. O’Neill et al., "A controlled trial of renal denervation for resistant hypertension," New England Journal of Medicine, vol. 370, no. 15, pp. 1393-1401, 2014.

[9] D. S. Atherton, N. L. Deep, and F. O. Mendelsohn, "Microanatomy of the renal sympathetic nervous system: a human postmortem histologic study," Clinical Anatomy, vol. 25, no. 5, pp. 628-633, 2012.

[10] P. S. Chen, L. S. Chen, M. C. Fishbein, S. F. Lin, and S. Nattel, "Role of the autonomic nervous system in atrial fibrillation: pathophysiology and therapy," Circulation Research, vol. 114, no. 9, pp. 1500-1515, 2014.

[11] J. Hellyer, A. Akingba, K. S. Rhee et al., "Autonomic nerve activity and blood pressure in ambulatory dogs," Heart Rhythm, vol. 11, no. 2, pp. 307-313, 2013.

[12] E. K. Choi, M. J. Shen, S. Han et al., "Intrinsic cardiac nerve activity and paroxysmal atrial tachyarrhythmia in ambulatory dogs," Circulation, vol. 121, no. 24, pp. 2615-2623, 2010.

[13] M. Chen, L. Yu, X. Zhou, Q. Liu, H. Jiang, and S. Zhou, "Lowlevel vagus nerve stimulation: an important therapeutic option for atrial fibrillation treatment via modulating cardiac autonomic tone," International Journal of Cardiology, vol. 199, pp. 437-438, 2015.

[14] Y. Hou, Q. Zhou, and S. S. Po, "Neuromodulation for cardiac arrhythmia," Heart Rhythm, vol. 13, no. 2, pp. 584-592, 2016.

[15] M. J. Shen, T. Shinohara, H. W. Park et al., "Continuous lowlevel vagus nerve stimulation reduces stellate ganglion nerve activity and paroxysmal atrial tachyarrhythmias in ambulatory canines," Circulation, vol. 123, no. 20, pp. 2204-2212, 2011.

[16] A. H. G. Driessen, W. R. Berger, S. P. J. Krul et al., "Ganglion plexus ablation in advanced atrial fibrillation: the AFACT study," Journal of the American College of Cardiology, vol. 68, no. 11, pp. 1155-1165, 2016.
[17] Z. Jiang, H. Yin, Y. He et al., "Efficacy and safety of novel epicardial circumferential left atrial ablation with pulmonary vein isolation in sustained atrial fibrillation," Heart Vessels, vol. 30, no. 5, pp. 675-681, 2015.

[18] G. Giannopoulos, C. Kossyvakis, C. Angelidis et al., "Coincidental ganglionated plexus modification during radiofrequency pulmonary vein isolation and post-ablation arrhythmia recurrence," Europace, vol. 19, no. 12, pp. 19671972, 2017.

[19] K. Nishida, T. Datino, L. Macle, and S. Nattel, "Atrial fibrillation ablation: translating basic mechanistic insights to the patient," Journal of the American College of Cardiology, vol. 64, no. 8, pp. 823-831, 2014.

[20] S. Zheng, Y. Zeng, Y. Li, J. Han, H. Zhang, and X. Meng, "Active ganglionated plexi is a predictor of atrial fibrillation recurrence after minimally invasive surgical ablation," Journal of Cardiac Surgery, vol. 29, no. 2, pp. 279-285, 2014.

[21] W. Nammas, J. K. Airaksinen, T. Paana, and P. P. Karjalainen, "Renal sympathetic denervation for treatment of patients with atrial fibrillation: reappraisal of the available evidence," Heart Rhythm, vol. 13, no. 12, pp. 2388-2394, 2016.

[22] L. Yu, B. Huang, Z. Wang et al., "Impacts of renal sympathetic activation on atrial fibrillation: the potential role of the autonomic cross talk between kidney and heart," Journal of the American Heart Association, vol. 6, no. 3, article e004716, 2017.

[23] B. Huang, L. Yu, B. J. Scherlag et al., "Left renal nerves stimulation facilitates ischemia-induced ventricular arrhythmia by increasing nerve activity of left stellate ganglion," Journal of Cardiovascular Electrophysiology, vol. 25, no. 11, pp. 1249-1256, 2014.

[24] W.-C. Tsai, C. Yi-Hsin, K. Chinda et al., "Effects of renal sympathetic denervation on the stellate ganglion and brain stem in dogs," Heart Rhythm, vol. 14, no. 2, pp. 255-262, 2017.

[25] Z. Jiang, Y. Zhao, A. Doytchinova et al., "Using skin sympathetic nerve activity to estimate stellate ganglion nerve activity in dogs," Heart Rhythm, vol. 12, no. 6, pp. 1324-1332, 2015.

[26] J. A. Armour, "Functional anatomy of intrathoracic neurons innervating the atria and ventricles," Heart Rhythm, vol. 7, no. 7, pp. 994-996, 2010.

[27] J. M. Cao, L. S. Chen, B. H. KenKnight et al., "Nerve sprouting and sudden cardiac death," Circulation Research, vol. 86, no. 7, pp. 816-821, 2000.

[28] B. L. Nguyen, M. C. Fishbein, L. S. Chen, P. S. Chen, and S. Masroor, "Histopathological substrate for chronic atrial fibrillation in humans," Heart Rhythm, vol. 6, no. 4, pp. 454-460, 2009.

[29] C. Gallo, P. P. Bocchino, M. Magnano et al., "Autonomic tone activity before the onset of atrial fibrillation," Journal of Cardiovascular Electrophysiology, vol. 28, no. 3, pp. 304-314, 2017. 


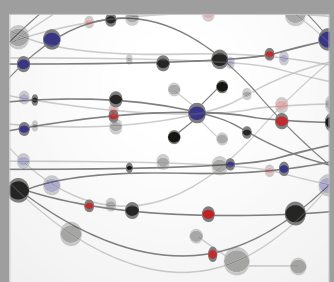

The Scientific World Journal
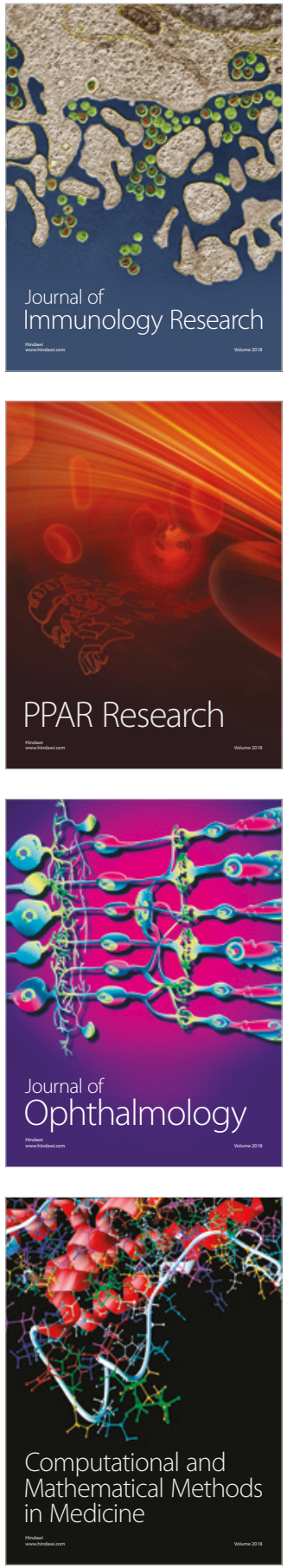

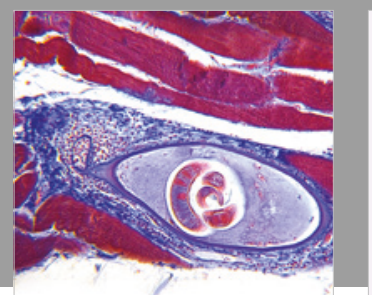

Gastroenterology Research and Practice

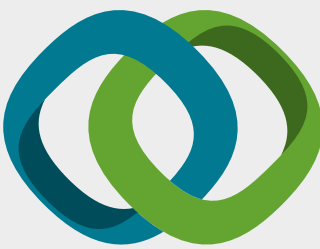

\section{Hindawi}

Submit your manuscripts at

www.hindawi.com
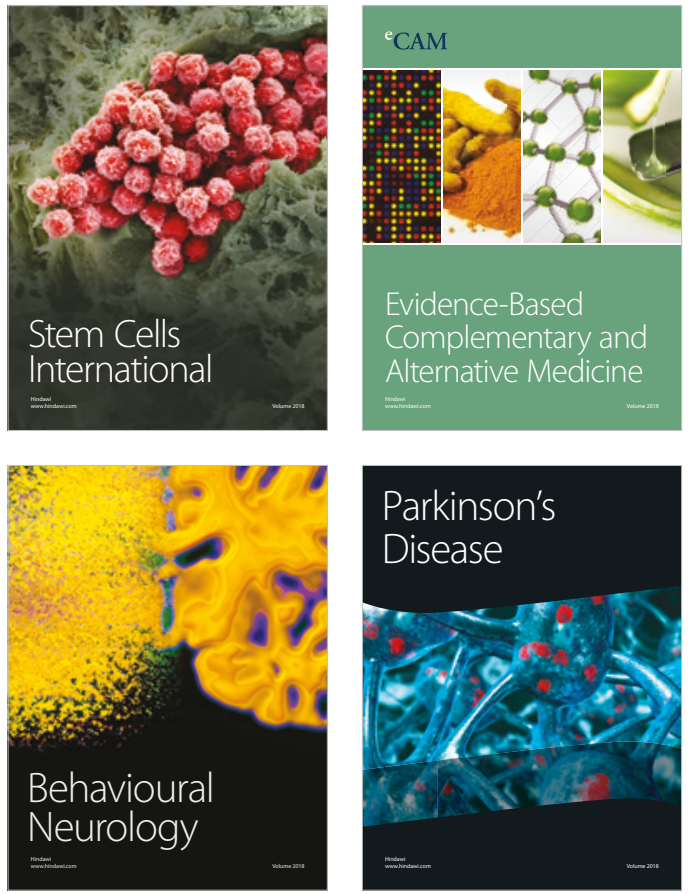

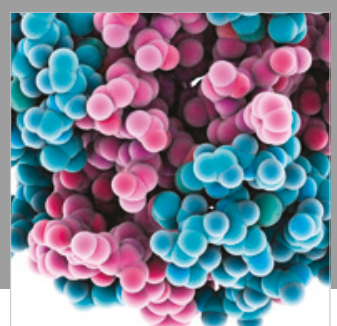

ournal of

Diabetes Research

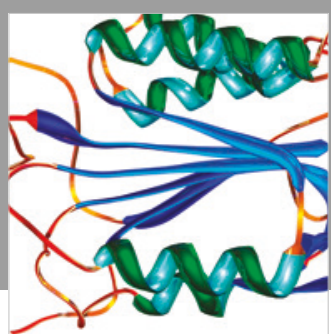

Disease Markers
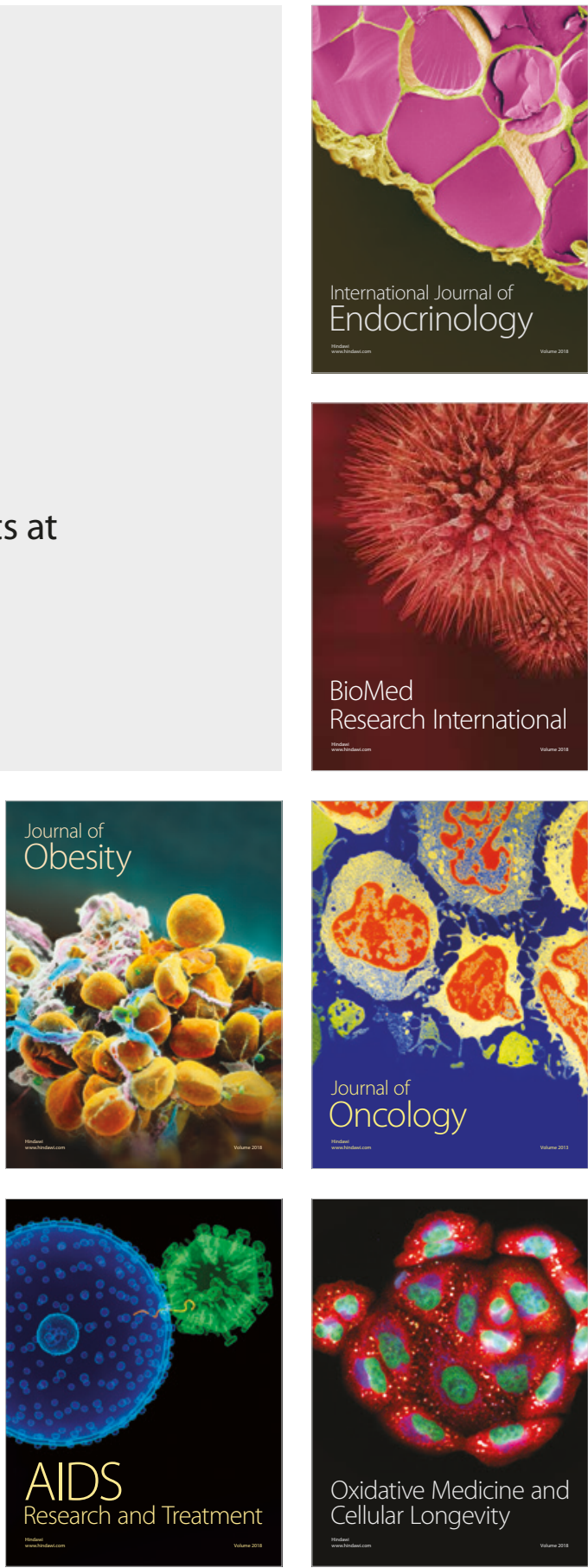